Solano, E., Arrieta, L., \& Mendoza, D. (2015). Comercio exportador sector textil - confecciones en Colombia y departamento del Atlántico: una aproximación a los modelos gravitacionales. Revista Lebret, 7. Bucaramanga, Colombia: Universidad Santo Tomás, pp. 79 - 100. ISSN: 2145-5996.

\title{
Comercio exportador sector textil - confecciones en Colombia y departamento del Atlántico: Una aproximación a los modelos gravitacionales*
}

\section{Export business in the textile and clothing industry in Colombia and its Atlantic Department: An approach to gravitational models}

\author{
Elcira Solano Benavides ${ }^{l}$ \\ Leonardo Arrieta Olascoaga ${ }^{2}$ \\ Dannyra Mendoza Cuello
}

\begin{abstract}
Resumen
El artículo analiza los factores que incentivan el comercio exportador del sector textil-confección en Colombia y en especial en el departamento del Atlántico. La metodología usada es el análisis de la teoría económica referente al comercio internacional. Además, se utilizan dos modelos gravitacionales para hallar los factores que determinan el comercio exportador del sector. Los resultados fueron: factores comunes entre socios como idioma, el país colonizador y el tamaño de la economía impulsan el sector. Los acuerdos comerciales no son determinantes para las exportaciones en el Atlántico; sin embargo, para Colombia es significativo estadísticamente.
\end{abstract}

Palabras clave

Sector textil-confección, economía internacional, tratados bilaterales y modelo gravitacional.

\section{Códigos de clasificación JEL: F00, F53, F12}

\begin{abstract}
The research studies the variables that encourage the export trading of the textile and clothing sector in Colombia and the department of the Atlántico. The methodology used is the analysis of international trade economic theory. Further, two gravity models are used for estimate the factors that determine the export the sector. The results are common factors among partners such as language; the colonizer country and the size of the economy drive the sector. Trade agreements are not decisive for exports in the Atlantic; however, for Colombia it is statistically significant.
\end{abstract}

\section{Keywords}

Textile and clothing sector, international economy, trade agreements and gravity model.

* El artículo deriva de la investigación "Competitividad comercial del sector textil en Colombia", apoyada institucionalmente por la Universidad del Atlántico.

1 Estudiante de Doctorado en Ciencias Económicas. Magíster en Economía. Profesora investigadora de la Universidad del Atlántico del grupo Economía de la Educación. Correo electrónico: elcirasolano@hotmail.com

2 Economista. Integrante del grupo Economía de la Educación, Universidad del Atlántico. Correo electrónico: leonardo.302008@hotmail.com.

3 Economista. Integrante del grupo Economía de la Educación, Universidad del Atlántico. Correo electrónico: dannyra_1@hotmail.com 


\section{Introducción}

El sector textil-confecciones en Colombia es importante en la economía colombiana por el número de empleos que genera; según el informe de la Superintendencia de Sociedades (2015), el sector para el 2014 representó el 9.2\% del PIB de la industria manufacturera colombiana, con 450 empresas textiles y 10000 de confecciones. El sector crea 200000 empleos directos y 600000 indirectos, no obstante $74 \%$ del empleo es no calificado. Además, el comportamiento del sector no es estable en la última década, por ejemplo, la contribución en el PIB manufacturero, su punto más alto fue en el 2007 con un 6,9\%. A partir de 2009 ha declinado su participación con $1,7 \%$, en 2010 con $4 \%$, el 2012 de $6 \%$ y el 2013 un 4\%. También, se observa que el sector es procíclico, es decir, mantiene la misma fluctuación que el PIB del país (Colombia), pero en las caídas del PIB son mayores en el sector, mostrando más vulnerabilidad.

Referente al comercio exportador, en el 2008 tenía unas exportaciones del orden de 580000 (cifra en miles de dólares FOB), en el 2009 esta cifra cae en un 50\% y no se recupera en los siguientes años. El informe de la Superintendencia de Sociedades (2013) explica que la caída de las exportaciones del sector se debe a cuatro puntos: los tratados bilaterales firmados por Colombia, la globalización de los mercados, el contrabando y a la utilización de empleo con baja remuneración en países asiáticos.

El presente artículo analiza el comercio exportador en el sector textil-confecciones en Colombia y en particular en el departamento del Atlántico, considerando las diferentes teorías de comercio internacional y utilizando la metodología econométrica gravitacional.

Este documento se encuentra organizado en las siguientes secciones: en la primera se presenta la introducción. En la segunda se analiza la teoría referente a la economía internacional. En la tercera se presenta la metodología utilizada para el análisis del comercio exportador del sector en Colombia y en el departamento del Atlántico. En la cuarta se muestran los resultados y discusiones del trabajo. Por último, se presentan las conclusiones.

\section{Discusión teórica}

A lo largo de la historia se han desarrollado teorías que explican el funcionamiento del comercio internacional y estudian los efectos de este sobre el bienestar, producción, consumo, precios, factores y distribución de recursos. Estas teorías se han caracterizado por mantener una serie de supuestos, desarrolladas cuando cambian los entornos económicos, sociales, políticos, comercial, entre otros. Smith (1776), con su teoría clásica de la ventaja absoluta, utiliza un único factor de producción trabajo; si un país usa este factor en la producción de un bien, y su costo total de producción en términos absolutos fuera inferior con respecto a los costos 
del otro país, entonces existe una ventaja absoluta para esta nación. El desarrollo en el comercio internacional replanteó la teoría de Smith (1776), originando la teoría de David Ricardo (1975). Esta teoría se fundamenta en la ventaja que ofrece la especialización en la elaboración de bienes y servicios, con el fin de reducir costes con respecto a otros países.

Complementando la teoría de David Ricardo (1975), Hecksher (1968) y Ohlin (1933), en el modelo de proporciones factorial explican que los países se especializan y exportan bienes en que su fabricación utiliza factores de producción abundante en el país. Además, importan bienes que utilizan para su fabricación factores escasos en el país. Por otro lado, se encuentra el modelo de competencia monopolística planteado por Krugman (1979); el modelo analiza el comercio basado en las economías de escala, partiendo de los problemas que presentan los mercados en competencia perfecta, mostrando qué firmas de gran tamaño oligopólicas tienen poder de mercado interno y externo con respecto a firmas que manejan bajo nivel de producción; las primeras tienen economías de escalas y poseen ventajas con respecto a costos, precios y calidad.

Cuando estos rendimientos crecientes de escala entran en juego con el comercio, los mercados se hacen, en general, imperfectamente competitivos. Krugman (1979) también plantea una política comercial internacional, apoyándose en los instrumentos usados por los gobiernos para influir en los intercambios comerciales a través de aranceles, cuotas de importación, subsidios y restricciones voluntarias a la exportación, barreras administrativas, libre comercio, economías de escala y mercados eficientes. A partir de las anteriores teorías, Krugman (1990) realiza nuevos planteamientos sobre la geografía económica y explica el aporte del comercio internacional al crecimiento de la economía en un entorno de países unificados por acuerdos y tratados. Pero también, con sectores de la industria concentrados en regiones que les da ventajas (ejemplo, costes de transportes) en el comercio internacional. Si el sector tiene incentivos para concentrarse en lugares específicos se le llama fuerzas centrípetas, pero si existen factores que hacen que las firmas se encuentren dispersas se dice que tienen fuerza centrífuga.

Por último, en términos del comercio internacional han surgido las políticas estratégicas comerciales en los acuerdos entre socios; es así que Spencer y Brander (1983) en su teoría de juegos, exponen que los Estados actúan de forma estratégica y diseñan políticas que por razones de bienestar para el país, subsidian ciertos sectores en la economía para que actúen en forma oligopólica y logren acaparar una cuota alta en el mercado internacional.

\section{Método}

Se considera un modelo panel data para determinar si el crecimiento del sector es significativo en las exportaciones. También se utilizó un modelo econométrico 
llamado gravitacional para determinar las variables que inciden en las exportaciones como: distancia, idioma, país colonizador, moneda, y el tamaño de la economía de los países socios.

La unidad de análisis de la investigación son las exportaciones del sector textil y confecciones en Colombia y del departamento del Atlántico.

El marco temporal del estudio son las exportaciones del sector en Colombia 20062013 y del departamento del Atlántico en los años comprendidos del 2006 al 2011.

Para estudiar la dinámica del sector se utilizó la base de datos de exportaciones para Colombia 2007-2013, disponible en la página Web del Departamento Administrativo Nacional de Estadística - DANE ${ }^{4}$. La información del departamento del Atlántico 2006-2011 se obtiene de la Dirección de Impuesto de Aduanas Nacionales - DIAN 5 .

De la base de datos de la Dian se filtraron las exportaciones en dólares de los socios comerciales del departamento del Atlántico, además se calcularon las tasas de crecimiento de las exportaciones por países y se estimó un modelo panel data para determinar si el crecimiento de las exportaciones está relacionado con los tratados bilaterales firmados por Colombia. De los datos del DANE, se filtraron las exportaciones por países del sector en Colombia y se calcularon las tasas de crecimiento de las exportaciones por socios y se estimó un modelo panel data para determinar si el crecimiento de las exportaciones por socios está relacionado con los tratados bilaterales firmados por Colombia. También se utilizaron datos del Banco Mundial y de la ONU para estimar un modelo gravitacional, que determina los factores que inciden en las exportaciones del sector para Colombia y el departamento del Atlántico.

\section{Resultados}

\section{Cadena productiva del sector textil-confecciones}

Figura 1. Cadena productiva del sector textil-Confecciones

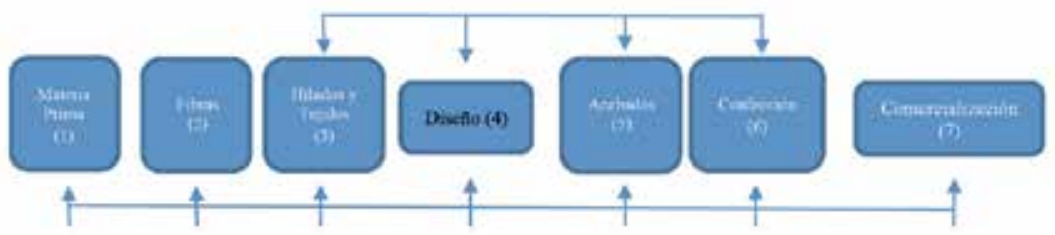

Fuente: Condo, A. et al. (2004).

http://www.dane.gov.co

5 La información a nivel nacional por parte del DANE no se ofrece en el mismo periodo de tiempo que para los departamentos del país. Esa es la razón de la utilización de la base de datos de la DIAN. 
La cadena tiene una estructura de siete etapas, las tres primeras relacionadas con la producción de materia prima, fibras, hilados y tejidos, estas etapas requieren mayor aporte de capital con nuevas tecnologías y personal altamente capacitado, es la etapa que produce mayor valor agregado y economías de escala. El proceso de acabados y confección es la etapa que requiere mayor cantidad de mano de obra no costosa y en la cual Colombia es fuerte. La comercialización es la última etapa que determina la producción hacia mercados nacionales o internacionales. Según cálculos de Castro, Contreras, Tamayo, \& Trujillo, (2013) las participaciones de las diferentes cadenas en el valor de producción, las confecciones lideran la participación: en 2001 representan el 79,5\% del valor de producción de la cadena, en 2011 su participación ascendió al $83,9 \%$. En el caso de la producción de algodón, pasó del $0,9 \%$ al 1,1\%. Por su parte, la cadena de hilos y textiles disminuyó su participación al pasar de 6,2\% y 13,4\% en 2001 , a $4,8 \%$ y $10,2 \%$ en 2011 , respectivamente.

Figura 2. Participación de las cadenas en el valor de la producción en el sector, 2011 Colombia

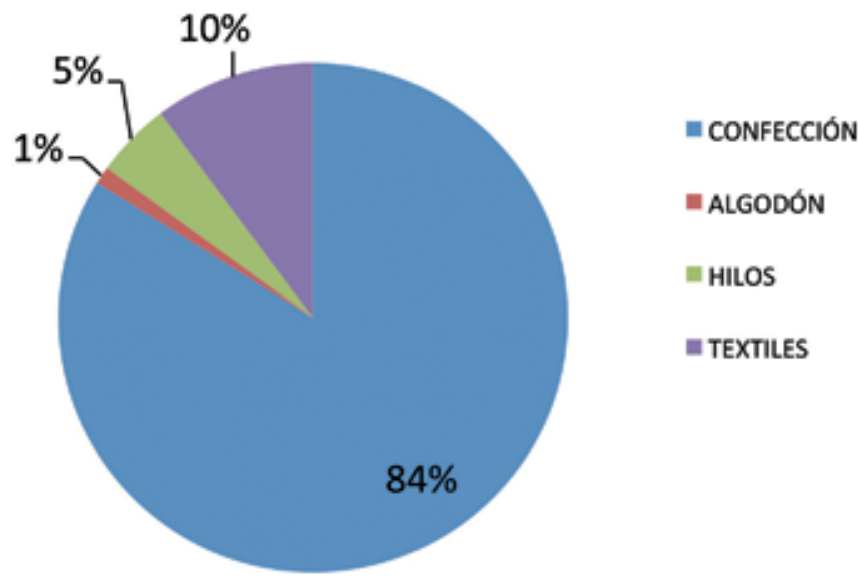

Fuente: DANE y cálculos de Fedesarrollo.

\section{Mercado del sector textil-confecciones en Colombia}

El sector se encuentra enmarcado en el modelo de competencia monopolística planteado por Paul Krugman (1979). Según datos de la Superintendencia de Sociedades en 2012, en Colombia, las grandes y medianas empresas del sector representan el 59\%. Cuando se consideran los ingresos operacionales o ventas de las grandes empresas que son 159 , tienen una cuota de mercado del $78 \%$ y las medianas el $18 \%$. Las pequeñas y microempresas que representan el $41 \%$ de las empresas tienen una cuota de mercado del $4 \%$. La concentración de los ingresos en las grandes y medianas empresas se debe a las economías de escala que se generan por fallos 
en el mercado. Es decir, barreras de entrada a capitales, tecnologías y asimetrías de información en este mercado.

Figura 3. Tamaño empresa del sector, Colombia, 2012

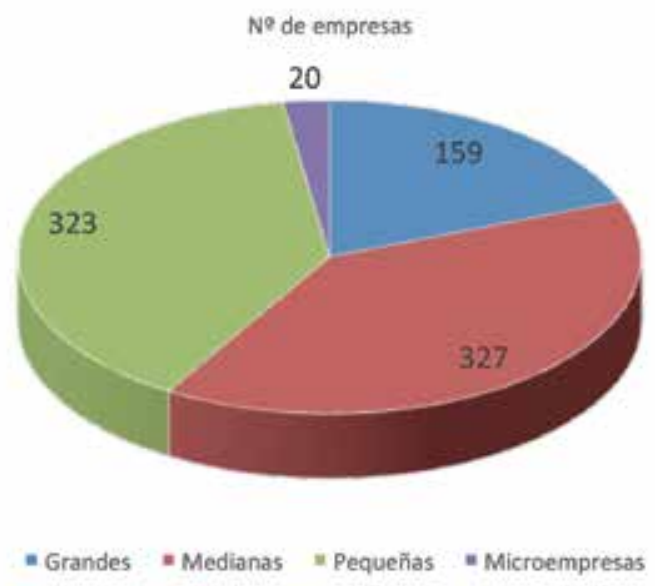

Fuente: Superintendencia de Sociedades.

Figura 4. Participación empresas en los ingresos operacionales del sector textilconfecciones, Colombia, 2012

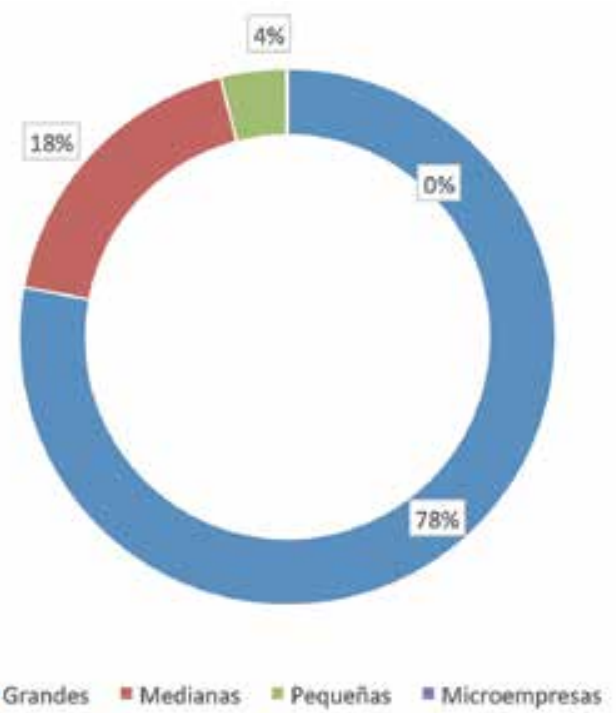

Fuente: Superintendencia de Sociedades, cálculos autores. 
Además, la teoría geografía económica de Krugman (1992) argumenta que las empresas tienden a tener incentivos y desincentivos para que una industria decida $\mathrm{o}$ no concentrarse en una región determinada. En Colombia existen incentivos de bajos costes de transporte para que el sector textil-confecciones se aglomere en el centro del país, por cercanía a los grandes mercados nacionales, así el 77\% de las empresas se aglomeran en Bogotá y Antioquia, entonces existen fuerzas centrípetas.

Tabla 1. Ubicación geográfica del sector textil-confecciones en Colombia, 2011

\begin{tabular}{l|r|r}
\hline \multicolumn{1}{|c|}{ Departamento } & Número de empresas & Participación \\
\hline Bogotá & 97803 & 48,61 \\
\hline Antioquía & 58006 & 28,83 \\
\hline Valle & 12132 & 6,03 \\
\hline Atlántico & 7283 & 3,62 \\
\hline Risaralda & 6318 & 3,14 \\
\hline Santander & 6076 & 3,02 \\
\hline Cundinamarca & 4125 & 2,05 \\
\hline Tolima & 2435 & 1,21 \\
\hline Norte de Santander & 2193 & 1,09 \\
\hline Caldas & 966 & 0,48 \\
\hline San Andrés y Providencia & 966 & 0,48 \\
\hline Cesar & 483 & 0,24 \\
\hline Nariño & 483 & 0,24 \\
\hline Bolívar & 241 & 0,12 \\
\hline Cauca & 241 & 0,12 \\
\hline Córdoba & 241 & 0,12 \\
\hline Huila & 241 & 0,12 \\
\hline Magdalena & 241 & 0,12 \\
\hline Meta & 241 & 0,12 \\
\hline Quindío & 241 & 0,12 \\
\hline Sucre & 241 & 0,12 \\
\hline Total & & \\
\hline & 241 & \\
\hline & 241 & \\
\hline
\end{tabular}

Fuente: DANE, cálculo autores.

\section{El sector textil-confecciones en Colombia y su entorno económico externo}

Según el DANE en el 2011, la industria textil representa el 12\% del PIB industrial, genera el 6,1\% del empleo manufacturo, el 5\% de las exportaciones en Colombia y es el sector con mayor participación en exportaciones no tradicionales. La tendencia de las exportaciones del sector en Colombia es la mayor participación en las confecciones. Colombia es un importador neto de textiles y exportador neto de confecciones, es decir, en la etapa del proceso productivo que requiere abundancia 
de capital el país es débil y por eso es importador neto de materia prima. En la etapa productiva de acabados y confección que tiene menor valor agregado es donde se tiene la fortaleza exportadora. Además, el sector presenta desventaja en términos de precios y calidad comparados con el mercado asiático, que la hace vulnerable en los tratados de libre comercio que el país ha firmado con varios países.

\section{Tendencia del mercado textil-confecciones internacional}

Asía tiene la mayor contribución en el mercado internacional, por ejemplo, según estadísticas del comercio internacional del 2012 y cálculos de los autores, las participaciones en exportaciones de prendas de vestir domina el mercado con un $58 \%$, en la cual China participa con el 37\%, Europa 30\% y América del sur y central con un 3\%. La política de Estado de China de fortalecer el sector con grandes inversiones para la producción de insumos y maquinarias, acompañado con mano de obra barata, ubica a este país en posición dominante en el mercado internacional.

Los países desarrollados han liderado la innovación de nuevos productos, nuevas tecnologías, con altos requerimientos en capital, además del liderazgo en cooperación internacional y comercial. Sin embargo, la tendencia en el mercado internacional es la política comercial de precios utilizada por China. Colombia es un país importador de materia prima, por ejemplo, el algodón y la fibra sintética que se produce en Colombia solo abastece el $35 \%$ de consumo nacional.

\section{Dinámica exportadora del sector textil y confecciones en Colombia y en el departamento del Atlántico}

El valor de las exportaciones en Colombia en el sector durante el periodo $2007-$ 2008 fue mayor al periodo 2009-2013. Las exportaciones del sector fueron afectadas por la crisis económica, reevaluación del tipo de cambio desde el 2008 y el cierre de relaciones comerciales con Venezuela en el 2009.

Figura 5. Crecimiento y comportamiento de las exportaciones del sector textilconfecciones en Colombia, periodo 2007-2013, total valor FOB en (USS)

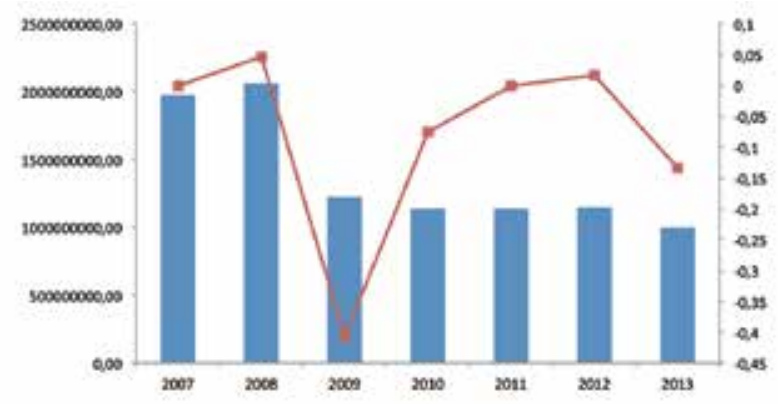

Fuente: DANE. 
Figura 6. Tipo de cambio bilateral, periodo 2007-2013

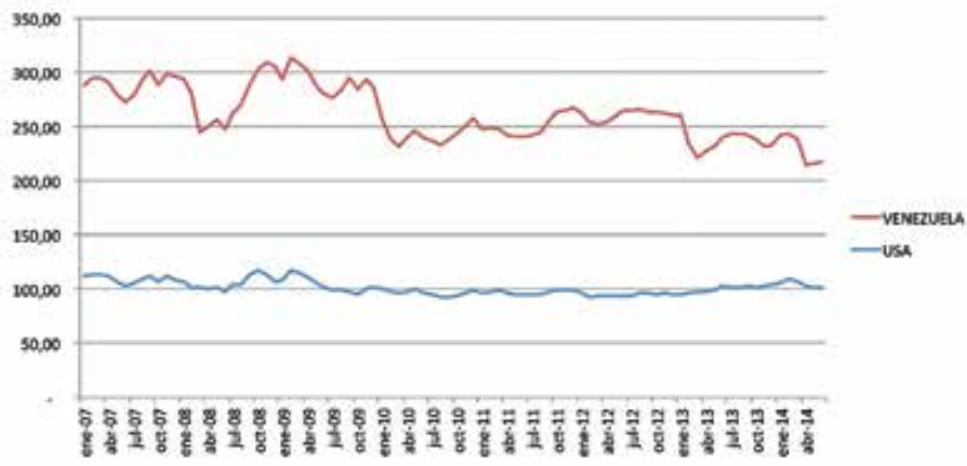

Fuente: Banco de la República.

La apreciación del peso colombiano frente al dólar durante el periodo de estudio afectó las exportaciones del sector, los precios con que opera no alcanzan su punto de equilibrio, considerando que los costos de producción y de distribución son altos en el sector.

Figura 7. Participación en exportaciones sector textil y confecciones por socio comercial, Colombia 2007-2013

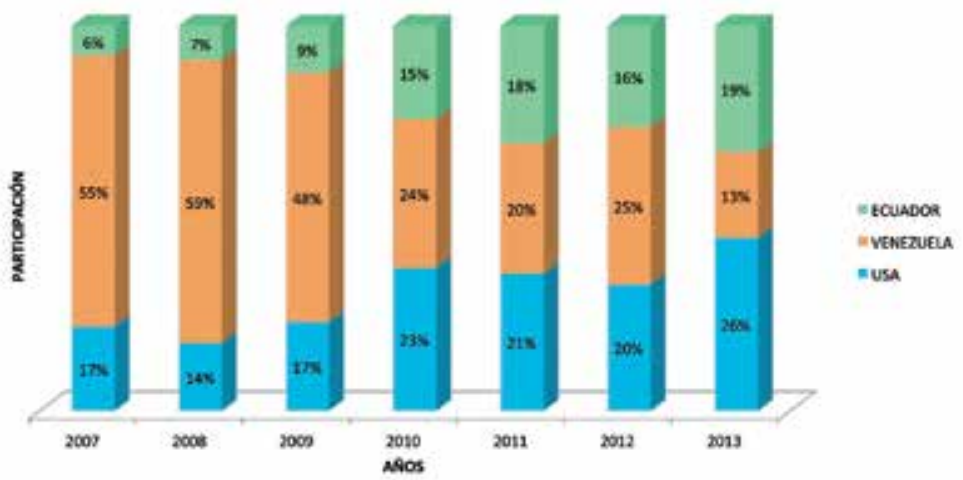

Fuente: DANE, cálculos de los autores. 
Figura 8. Comportamiento de las exportaciones sector textil-confecciones en el departamento del Atlántico, periodo 2006-2011, total valor FOB en (USS)

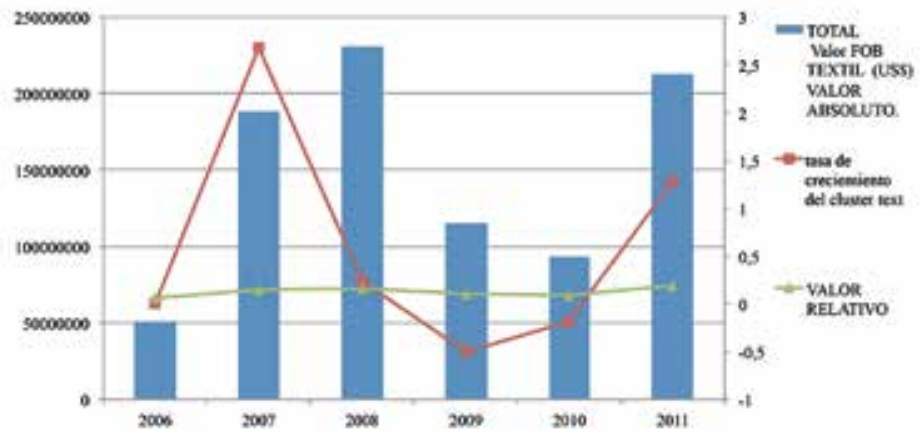

Fuente: DIAN y cálculo autores.

Considerando los socios que tienen mayor participación, se observa que son: Estados Unidos y Ecuador, mientras Venezuela sigue disminuyendo durante todo el periodo. Las exportaciones en el departamento del Atlántico han experimentado una mayor participación en las exportaciones totales del departamento, en el 2006 tenía una participación del 5.5\%, en el 2011 pasó a 18.8\%. Las exportaciones de la industria textil pasaron de ser USD \$51 179 815,3 millones en el 2006, a USD \$ 389145 522,6 millones en el 2011.

Tabla 2. Participación de los 10 principales socios comerciales del clúster textil en el departamento del Atlántico, exportaciones periodo 2006-2011

\begin{tabular}{l|c}
\hline \multicolumn{1}{c|}{ PAÍS } & PARTICIPACIÓN \\
\hline Venezuela & $20,21 \%$ \\
\hline Estados Unidos & $12,11 \%$ \\
\hline Corea & $6,85 \%$ \\
\hline China & $4,85 \%$ \\
\hline Ecuador & $3,37 \%$ \\
\hline México & $2,10 \%$ \\
\hline Brasil & $1,94 \%$ \\
\hline Panamá & $1,62 \%$ \\
\hline Alemania & $1,43 \%$ \\
\hline Dinamarca & $1,33 \%$ \\
\hline
\end{tabular}

Fuente: DIAN y cálculo autores. 


\section{Competitividad exportadora del sector en Colombia}

Según Latruffe (2010) la ventaja en el comercio internacional de un mercado se encuentra en la variedad y calidad de los productos, los tratados de libre comercio y la tasa de cambio. Krugman (1999) considera que las empresas de competencia imperfectas tipo oligopolio tienen ventajas en el comercio internacional, como también la ubicación geográfica de las empresas les da ventaja: cerca de los grandes mercados nacionales y a los puertos marítimos. Oral, Kettani, \& Yolalan (1992) consideran que la ventaja del mercado en el sector externo depende de los precios del mercado. También el indicador de ventaja comparativa revelada indica el desempeño del país como exportador de la producción del sector; se estima como el cociente entre la proporción de las exportaciones de un país con respecto al mercado mundial y la proporción del mercado del país en las exportaciones Castro et al. (2013); el indicador para el 2011 en Colombia son: algodón 0,05, para hilados de algodón es de 0,09 y textiles 0,886 ; donde indicadores por debajo de cero muestran menor ventaja y cercano a 1, mayor. Según el mismo informe la productividad laboral es creciente para el periodo 2008-2012 que hace referencia a mejores tecnologías y mayor producción por trabajador: en la cadena de producción de hilados y tejidos se observan las mayores productividades y en confecciones es menor. Analizando el sector desde la perspectiva de la economía geográfica como lo plantea Krugman (1999); las empresas exportadoras del sector tienden a ubicarse estratégicamente, de tal manera que sus costos de producción se reduzcan, y así obtienen un mayor beneficio. Este fenómeno origina un aglomeramiento de empresas con el fin de aprovechar las ventajas geográficas e incursionar en el mercado internacional.

Las exportaciones en Colombia para el 2011 las lideran las empresas ubicadas en Antioquía, con un $31 \%$, sigue Bogotá $11.9 \%$, Atlántico $4 \%$, Cali $1,3 \%$ y Santander $1 \%$. El sector en Colombia presenta en su producción aglomeramiento en la parte central del país, donde se encuentran los grandes mercados nacionales. Sin embargo, cuando incursionan en el mercado externo se encuentran con costos elevados de transporte, en el sentido de que las empresas con perfil exportador se encuentran en Antioquía, ubicado lejos de los puertos marítimos. Por ejemplo, el costo de transporte al puerto más cercano Cartagena tiene una distancia en ruta de 637 kilómetros, a precios de 2013 un contenedor cuesta 1000 dólares y el precio del costo de transporte a Hamburgo Alemania es de 1100 dólares.

Cuando se estudia el departamento del Atlántico, se observa que las empresas textiles en el departamento están ubicadas estratégicamente en Barranquilla que tiene cercanía a la frontera con Venezuela y un puerto marítimo internacional que lo conecta a Estados Unidos; dos socios estratégicos que suman el $34 \%$ de las exportaciones del sector en el Atlántico. El departamento cuenta con 441 empresas exportadoras, de las cuales solo cinco manejan una mayor participación del mercado internacional, estas son industrias Cannon con una participación de (36\%), le sigue Avianca Continente Americano Aerovías S.A con un (7.8\%), Dist plex SACI (5,6\%), 
Saafartex S.A. (5,5\%) Impos y Representaciones Layan-Ahmad Mohammad (4,9\%) la sumatoria de estas empresas conforman el 59,9\% del mercado. Se observa, que dos grandes empresas participan en el mercado internacional con cerca del $50 \%$ de las exportaciones del sector en el departamento. Aproximándose a la teoría de Krugman (1999), las grandes empresas con acceso a tecnologías y capital tienen economías de escala para ser competitivas en el sector a nivel de costes.

Tabla 3. Empresas exportadoras de la industria textil y confecciones en Colombia, 2011

\begin{tabular}{|c|c|c|}
\hline EMPRESA & $\begin{array}{l}\text { PARTICIPACIÓN } \\
\text { EXPORTACIONES }\end{array}$ & UBICACIÓN GEOGRÁFICA \\
\hline Textiles Fabricato S.A. & $6,4 \%$ & Antioquía \\
\hline Enka de Colombia S.A. & $5,9 \%$ & Antioquia \\
\hline C.I. Jeans S.A. & $5,2 \%$ & Antioquía \\
\hline Industrias Cannon Colombia S.A. & $4, \%$ & Atlántico \\
\hline Manufacturas Eliot S.A. & $4 \%$ & Bogotá \\
\hline C.I. Netcap S.A. & $3,2 \%$ & Antioquia \\
\hline Vestimundo S.A. & $3 \%$ & Antioquia \\
\hline C.I. Dugotex S.A. & 2,5 & Santander \\
\hline Compañía Colombiana de Tejidos S.A. Coltejer & $2,4 \%$ & Antioquía \\
\hline Lafayette S.A. & $1,9 \%$ & Bogotá \\
\hline Comercializadora Internacional Expofaro S.A. & $1,8 \%$ & Antioquia \\
\hline Supertex S.A. & $1,7 \%$ & Antioquia \\
\hline Permoda S.A. & $1,5 \%$ & Bogotá \\
\hline Protela S.A. Altex & $1,5 \%$ & Bogotá \\
\hline Fábrica de Calcetines Crystal S.A. & $1,3 \%$ & Antioquía \\
\hline Studio F. Internacional Fashion Corporación S.A. & $1,3 \%$ & Cali \\
\hline Vital Jeans S.A. C. I. & $1,1 \%$ & Bogota \\
\hline Industria Colombiana de Confecciones S.A. & $1,0 \%$ & Pereira \\
\hline Encajes S.A. Colombia & $1,9 \%$ & Bogotá \\
\hline Otras & $48,1 \%$ & \\
\hline
\end{tabular}

Fuente: DIAN y cálculo de los autores. 
Tabla 4. Participación de las 10 principales empresas exportadoras del sector textil-confecciones en el departamento del Atlántico, 2006-2011

\begin{tabular}{l|c}
\multicolumn{1}{c|}{ EMPRESAS } & Participación \\
\hline Inds Cannon de Colombia S. A. & $35,66 \%$ \\
\hline Avianca Aerovías Continente Americano S.A. & $7,82 \%$ \\
\hline Dist. Plex S. A. S. C. I. & $5,60 \%$ \\
\hline Saafartex S. A. & $5,45 \%$ \\
\hline Imps. y Representaciones Layan - Ahmad Mohammad & $4,91 \%$ \\
\hline France Sport Wear International de Colombia S. A. & $2,81 \%$ \\
\hline Ensacar S. A. & $2,60 \%$ \\
\hline Jacadi de Colombia S. A. Antes Jacadi de Colombia Ltda. & $2,57 \%$ \\
\hline Ejército Nacional Contaduría Principal Comando Ejército & $2,34 \%$ \\
\hline Fca. de Hilazas Vanylon S. A. & $2,22 \%$ \\
\hline
\end{tabular}

Fuente: DIAN y cálculo de los autores.

\section{Análisis del sector textil en los acuerdos bilaterales}

Lo expuesto sobre la dinámica exportadora del sector, los principales socios del país y el departamento son Venezuela y EE. UU., seguidos por Corea, China, Ecuador, México, Brasil y Panamá. De lo anterior se puede deducir que, aunque Colombia actualmente tiene acuerdos comerciales bilaterales vigentes con Chile, Canadá, México y EE. UU., se observa que solo este último tiene una gran participación (12.1\%) en las exportaciones del departamento 2011 y en Colombia de 26\% para el 2013. De lo anterior se deduce que las exportaciones del sector no dependen únicamente de las políticas comerciales establecidas en los tratados, pero otros factores pueden afectar las exportaciones del sector: la inversión, aplicación de nuevas tecnologías, la economía de escala en las empresas, la estrategia de política de Estado en el sector, la política comercial con socios estratégicos como Venezuela, el tipo de cambio y los costos de transportes en el sentido que gran parte del mercado exportador de Colombia se encuentran a grandes distancias de los puertos internacionales.

En los distintos acuerdos bilaterales que tiene Colombia, los textiles no se clasifican netamente como una mercancía de origen por depender de materias primas importadas. Se plantean políticas comerciales estratégicas sujetas a ciertas reglas de origen y preferencias arancelarias, entre las cuales se destacan: la posibilidad que tienen las partes de pedir la modificación de una norma de origen si el insumo respectivo para la elaboración del tejido o la confección no se encuentra en cantidades comerciales. También se debe considerar la posibilidad que los tejidos o confecciones hechos a mano o con telares manuales o mercancías folclóricas tengan acceso 
inmediato y libre de aranceles. Establecer una lista de insumos que no se encuentran en cantidades comerciales y sirven para producir textiles y confecciones, gozando de algunas preferencias de origen con el fin de facilitar el comercio de estos productos.

\section{Análisis econométrico del sector textil-confecciones}

\section{Modelo panel data de crecimiento de las exportaciones}

El modelo determina si el crecimiento de las exportaciones de los socios comerciales en el sector está relacionado con los acuerdos bilaterales firmados por Colombia.

$$
Y_{i t}=v_{i}+\beta_{1} X_{1 i t}+e_{i t}
$$

Donde $v_{i}$ es un vector de variables dicotómicas para cada socio, $\beta_{1}=$ tasa de crecimiento del i-esimo socio comercial.

Tabla 5. Resultados del modelo crecimiento panel data

\begin{tabular}{|c|c|c|c|c|c|c|c|}
\hline \multicolumn{4}{|c|}{ Modelo departamento del Atlántico } & \multicolumn{4}{|c|}{ Modelo Colombia } \\
\hline Socios comerciales & Coeficientes & $\begin{array}{l}\text { Error } \\
\text { estándar }\end{array}$ & $\mathrm{p}>[\mathrm{z}]$ & $\begin{array}{c}\text { Socios } \\
\text { comerciales }\end{array}$ & Coeficientes & $\begin{array}{c}\text { Error } \\
\text { estándar }\end{array}$ & $\mathrm{p}>[\mathrm{z}]$ \\
\hline $\begin{array}{c}\text { Tasa de } \\
\text { crecimiento }\end{array}$ & $3,33 \mathrm{e}-13$ & $3,27 e+16$ & 0 & $\begin{array}{c}\text { Tasa de } \\
\text { crecimiento }\end{array}$ & 0,204580 & 0,8017 & 0,013 \\
\hline Alemania & 2,441648 & 0,17224 & 0 & Venezuela & $-2,61431$ & 0,3718 & 0,00 \\
\hline Angola & $-5,03215$ & 1,5598 & 0 & Perú & 2,8460 & 0,3648 & 0,00 \\
\hline Antigua y Barbuda & $-1,22263$ & 0,2247 & 0,001 & Alemania & 3,3744 & 0,3646 & 0,00 \\
\hline Antilla Holandesa & $-5,28089$ & 0,18418 & 0 & Países Bajos & $-0,05459$ & 0,3646 & 0,882 \\
\hline Argentina & $-1,64889$ & 0,5808 & 0 & Japón & $-2,66899$ & 0,3811 & 0,000 \\
\hline Australia & $-2,55400$ & 0,9251514 & 0,005 & Francia & 2.5198 & 0,4276 & 0,000 \\
\hline Bahamas & $-4,67373$ & 1,40723 & 0,006 & Bélgica & $-1,1200$ & 0,3707 & 0,004 \\
\hline Bangladesh & $-5,74094$ & 2,167276 & 0,001 & Ecuador & 1,9419 & 0,3649 & 0,000 \\
\hline Chile & $-1,86487$ & 0,6514009 & 0,008 & México & 3,8437 & 0,3647 & 0,000 \\
\hline China & 2,260682 & 0,1591049 & 0,004 & & & & \\
\hline Corea del Sur & $-5,73156$ & 1,40355 & 0 & & & & \\
\hline Costa de Marfil & 2,099819 & 0,4431538 & 0 & & & & \\
\hline Cuba & $-2,00500$ & 0,5367696 & 0 & & & & \\
\hline Dinamarca & 4,53417 & 0,1863973 & 0 & & & & \\
\hline Dominicana & $-9,05456$ & 0,3904661 & 0 & & & & \\
\hline El Salvador & $-2,65027$ & 0,5717479 & 0 & & & & \\
\hline España & $-4,94457$ & 0,8095311 & 0 & & & & \\
\hline Francia & $-1,89175$ & 0,3350649 & 0 & & & & \\
\hline Gahana & $-2,56164$ & 0,6879328 & 0 & & & & \\
\hline Groenlandia & $-3,58853$ & 1,058746 & 0,001 & & & & \\
\hline
\end{tabular}




\begin{tabular}{l|r|r|r|r|l|l|l}
\hline Grecia & $-6,01996$ & 1,443398 & 0 & & & & \\
\hline Guatemala & $-4,06012$ & 0,2164305 & 0 & & & & \\
\hline Guyana & $-4,24892$ & 0,7430301 & 0 & & & & \\
\hline Haití & $-2,56408$ & 0,2564492 & 0 & & & & \\
\hline Honduras & 5,922001 & 2,806961 & 0,035 & & & & \\
\hline Hong Kong & $-3,96875$ & 0,9610179 & 0 & & & & \\
\hline Indonesia & $-1,08402$ & 0,3226051 & 0,001 & & & & \\
\hline Irlanda & 2,27273 & 0,1841918 & 0 & & & & \\
\hline Israel & 1,282645 & 0,381779 & 0,001 & & & & \\
\hline Jamaica & $-4,42008$ & 0,3659405 & 0 & & & & \\
\hline Líbano & 0,656387 & 0,1931241 & 0,001 & & & & \\
\hline Martinica & $-4,91147$ & 0,1546581 & 0 & & & & \\
\hline Nicaragua & $-4,88529$ & 1,498143 & 0,001 & & & & \\
\hline Noruega & 4,610029 & 0,3943082 & 0 & & & & \\
\hline Nueva Zelandia & $-1,93617$ & 0,806475 & 0,016 & & & & \\
\hline Consolidado & 12,74799 & 0,0793312 & 0 & & 16,00463 & 0,2579 & 0,000 \\
\hline
\end{tabular}

Fuente: Elaboración propia.

La tasa de crecimiento de las exportaciones del sector textil en ambos modelos es significativa. De los 91 socios comerciales del departamento solo resultaron significativos 35. Para el modelo de Colombia se escogieron los socios que tienen mayor participación en las exportaciones, por ello todos resultaron significativos.

Para el departamento del Atlántico, solo las tasas de crecimiento de las exportaciones con Chile, que tiene tratado bilateral vigente para el periodo de estudio, resultaron significativas, pero su impacto fue negativo sobre las exportaciones en el departamento del Atlántico (-1,86487), los otros socios comerciales que tienen firmados tratados bilaterales con Colombia no resultaron significativas sus tasas de crecimiento.

El modelo para Colombia en el periodo de estudio 2007-2013, solo la tasa de crecimiento de México con el que se tiene tratado bilateral, fue significativa y su impacto es positivo $(3,8437)$.

En el modelo del departamento del Atlántico, las tasas de crecimiento de socios que resultaron significativas con impacto positivo sobre las exportaciones a pesar de no contar con tratados de libre comercio son Alemania $(2,44)$, China $(2,260)$, Costa de Marfil (2,09), Dinamarca $(4,63)$, Honduras $(5,92)$, Irlanda $(2,27)$, Israel $(1,28)$, Líbano $(0,65)$ y Noruega $(4,61)$; se puede apreciar que estos socios comerciales por el factor geográfico están lejanos al puerto Marítimo de Barranquilla; que se encuentra en el departamento del Atlántico. El socio comercial Venezuela en el Atlántico, no sale significativo a pesar de que geográficamente es cercano al departamento. 
Para el modelo de Colombia, se observa que el socio Venezuela es significativo en las exportaciones, pero tiene un impacto negativo sobre las exportaciones del sector $(-2,61)$, parece que el rompimiento de relaciones comerciales con el socio afectó negativamente las exportaciones. Los otros socios que afectaron negativamente las exportaciones fueron países bajos $(-0,05459)$, Japón $(-2,66)$ y Bélgica $(-1,12)$. Los socios que impactan positivamente las exportaciones del sector son; Alemania $(3,3744)$, Francia $(2,51)$ y Ecuador $(1,9419)$; solo el último socio tiene frontera con Colombia.

\section{Modelos gravitacionales}

Los modelos gravitacionales, llamados así por la relación que se da en el comercio internacional entre países en función directamente proporcional al (PIB), tamaño del país e inversamente proporcional a la distancia entre países. Los modelos buscan encontrar los determinantes del comercio internacional utilizando los flujos comerciales en grupos de países que tienen características comunes. Tinbergen (1962) y Pöyhönen (1963) estimaron una serie de modelos gravitacionales de flujos comerciales bilaterales y hallaron que el volumen de transacciones comerciales internacionales aumentaba en la medida que se incrementaba el PIB real o per cápita de las economías y, por el contrario, disminuía con el aumento de los costos de transporte entre los países socios, medido esto por la distancia bilateral entre estos. Además, por los grandes consumos, mejores tecnologías y mejores infraestructuras que tienen los países desarrollados, se espera que los países con menor PIB tengan mayor comercialización con estos países. Otra variable por considerar es el costo de transporte de los bienes que se comercializan y es por ello que se espera que a mayor distancia disminuya el comercio entre los países socios. Otras variables que se le han agregado al modelo son las características comunes que comparten los socios comerciales, por ejemplo la población, la cercanía geográfica, es decir, si comparten fronteras o si tienen un idioma en común o si fueron colonizados por el mismo país.

Especificación del modelo

$$
\begin{aligned}
& \text { LnX }_{i t}=\beta_{o}+\operatorname{lnpib}_{i t}+\text { Inpibpercapita }_{i t}+\operatorname{lnpoblación}_{i t}+\operatorname{lnareakm}_{i t}^{2} \\
& + \text { Indistanciakm }_{i t}+\text { idioma }_{i j}+\text { acessomaritimo }_{i t}+\text { colonizado }_{i t} \\
& + \text { monedacomún }_{i t}+\text { acuerdos }_{i t}+\mu_{i t}
\end{aligned}
$$

\section{Dónde:}

$i$ : indica las exportaciones del sector del socio $i$ con el departamento del Atlántico

$t$ : las exportaciones en el tiempo con los socios $i$.

$L n X_{i t}=$ logaritmo de las exportaciones del sector del socio $i$ con el departamento del Atlántico en el tiempo $t$ 
$\ln p i b_{i t}=$ logaritmo del pib real del socio $i$ en el tiempo $t$.

$\operatorname{lnpibpercapita~}_{i t}=$ logaritmo del pib per cápita del socio $i$ en el tiempo $t$.

Inpoblación = logaritmo de la población del socio $i$ en el tiempo $t$

$\operatorname{lnareakm}_{i t}^{2}=$ logaritmo del área al cuadrado del socio $i$ en el tiempo $t$

Indistanciakm ${ }_{i t}=$ logaritmo de la distancia del socio i en el tiempo $t$.

idioma $_{i j}=$ variable dummy toma valor 1 si se tiene un idioma en común con el socio $i$ o 0 si no lo tiene.

acessomaritimo $_{i t}=$ variable dummy toma valor 1 si el socio tiene acceso marítimo o 0 si no lo tiene.

colonizado $o_{i t}=$ variable dummy toma valor 1 si el socio fue colonizado por el mismo país que Colombia o 0 si no fue colonizado.

monedacomún $_{i t}$ variable dummy toma valor 1 si el socio tiene moneda común con Colombia o 0 si no lo tiene.

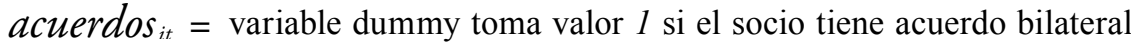
con Colombia o 0 si no lo tiene.

Tabla 6. Resultados del modelo gravitacional

\begin{tabular}{c|c|c|c|c|c|c|c}
\hline \multicolumn{4}{c|}{ Modelo departamento del Atlántico } & \multicolumn{4}{c}{ Modelo Colombia } \\
\hline Variables & Coeficientes & $\begin{array}{c}\text { Error } \\
\text { estándar }\end{array}$ & $\mathrm{P}>$ lzl & Variable & Coeficientes & $\begin{array}{c}\text { Error } \\
\text { Estándar }\end{array}$ & $\mathrm{P}>$ lzl \\
\hline LnPIB & 1,049807 & 0,0585641 & 0 & LnPIB & 2,2438 & 0,6597 & 0,001 \\
\hline $\begin{array}{c}\text { Ln } \\
\text { distancia }\end{array}$ & $-1,429838$ & 0,1838332 & 0 & $\begin{array}{c}\text { Ln área } \\
\mathrm{km}^{2}\end{array}$ & $-0,8706$ & 0,4657 & 0,062 \\
\hline Colonizador & 2,038834 & 0,2181725 & 0 & $\begin{array}{c}\text { Ln } \\
\text { distancia }\end{array}$ & $-3,5339$ & 0,6345 & 0,000 \\
\hline & & & & Idioma & 4,451 & 1,7258 & 0,010 \\
\hline & $-3,649184$ & 1,346885 & 0,007 & Cons & 0,6451 & 9,7632 & 0,496 \\
\hline
\end{tabular}

Fuente: Elaboración propia.

En ambos modelos aplicados los test, los mejores fueron los de efectos aleatorios con los siguientes resultados. Para el departamento del Atlántico: un aumento del 1\% en el PIB de los países socios genera una variación en igual sentido del 1,049\% en las exportaciones del sector. La distancia que hace relación a los costos de transportes es una variable significativa, es decir, un aumento del 1\% en kilómetros cuadrados con el país socio disminuye las exportaciones en este sector en $-0.87 \%$. 
Tener en común el país que los colonizó entre socios comerciales es significativo en la generación del comercio del sector, en otras palabras el ser colonizado por el mismo país aumenta las exportaciones en $2.039 \%$, en este modelo los tratados comerciales no son significativos. En el modelo gravitacional para Colombia: de igual forma, cuando el PIB aumenta en 1\%, las exportaciones de los países socios en textiles aumentan en $2,24 \%$. La distancia es una variable significativa en el modelo, es decir, un aumento del 1\% en kilómetros cuadrados con el país socio, disminuye el comercio en este sector en $-3,53 \%$. El área cuadrada en este modelo es significativa por estar asociada con el tamaño del socio comercial, un aumento en área en $1 \%$ en kilómetro cuadrado, las exportaciones disminuyen en $-0,87 \%$. Tener en común el idioma de los socios (español) es significativo en la generación del comercio de este sector en (4,51\%). Para Colombia los acuerdos comerciales entre socios sí es significativo; es decir, si se tiene acuerdo comercial las exportaciones aumentan en $(1,67 \%)$. Comparando ambos modelos, el departamento del Atlántico tiene un puerto marítimo internacional en Barranquilla, es por ello que el impacto negativo de la distancia es menor que el modelo de Colombia. El PIB en ambos modelos es importante y aumentan las exportaciones. Los factores comunes como idioma y colonizador impulsan el comercio en textiles; además que los socios con idioma y colonizador común están muy cercanos en distancia a Colombia. Los acuerdos comerciales no son relevantes para las exportaciones en el departamento, porque está cercano a un puerto marítimo; sin embargo, cuando es Colombia, las empresas exportadoras que se encuentran en Bogotá y Medellín parte central del país y lejos de los puertos marítimos; dependen más de los incentivos de los acuerdos comerciales.

\section{Discusión de los resultados econométricos}

En relación con los aportes teóricos y los resultados obtenidos en los análisis de la investigación, se hace una discusión considerando los resultados de diferentes trabajos en el artículo. Jiménez, Martínez, Mora, \& Martínez (2004) encontraron que países considerados socios comerciales con México, avalados por acuerdos comerciales presentan una tendencia contraria al acercamiento comercial. Para el caso del presente trabajo para el sector en el departamento del Atlántico, los acuerdos comerciales bilaterales vigentes al 2011 son: Chile, Canadá, México y EE. UU. Sin embargo, solo Chile presenta tasas de crecimientos negativas en exportaciones significativas; se puede decir que no necesariamente los acuerdos comerciales traen como consecuencia acercamientos comerciales en el sector. Para Colombia los acuerdos sí son importantes para las exportaciones de la industria. El aporte del trabajo es que se realizó a un sector específico y además de Colombia en un departamento, Atlántico.

Referente al modelo gravitacional se espera que los países más grandes comercien más en relación con los pequeños, debido, entre otros aspectos, al tamaño de sus economías. El PIB, que se considera como una proxy del tamaño económico, influye 
de forma positiva sobre la magnitud de las exportaciones. Para Díaz (2012), en el modelo gravitacional para Colombia sobre las exportaciones de todos los sectores, el PIB colombiano y de los países socios, resultaron significativas y positivas como lo dice la teoría. Igual en el trabajo de Bacaria, Osorio y Artal (2013), en el modelo gravitacional el PIB resulta ser el factor más importante en la determinación de los flujos comerciales, ya que ejerce un fuerte impacto positivo sobre el flujo de las exportaciones. Igual para el sector textil-confección se encuentra que el impacto de las economías grandes con su indicador PIB influye positivamente sobre las exportaciones del sector. La distancia debe presentar una elasticidad negativa, lo que indicaría que a mayor distancia el impacto sobre las exportaciones son menores; lo anterior por el aumento del costo de transporte. En Bacaria et al. (2013), este factor fue negativo y con valor a uno. Para el presente trabajo la elasticidad es negativa y mayor a uno, indicando que los costos de transportes es un factor negativo para las exportaciones. Para Cárdenas y García (2004) el impacto de la distancia es mayor en la medida que pasa el tiempo; en 1950 es de $-0,75$, en los ochenta es $-1,4$, indicando el impacto de los costos de trasporte aumentan con el tiempo en Colombia. Para Díaz (2011), si la distancia entre Colombia y sus socios aumenta en 1\%, sus exportaciones disminuyen en $1,34 \%$.

La variable vínculo colonial hace referencia al comercio que se realiza con países que comparten el mismo país colonizador. En Bacaria et al. (2013) la variable vínculo colonial es significativa y positiva, para el sector textil-confecciones este factor es igual de significativo. Lo anterior, es posible que se dé porque los países que tienen el mismo vínculo colonial son latinoamericanos que se encuentran en el mismo continente y como la distancia también es un factor favorable para las exportaciones. López y Muñoz (2008) estimaron un modelo gravitacional para los países de Chile y México, donde la variable distancia es significativa para ambos países y tiene mayor peso en México que en Chile; por el gran flujo comercial que tiene con USA. El valor del coeficiente asociado a los acuerdos comerciales es diferente para ambos países, en Chile se comporte mejor los TLC que en México. La variable que es significativa en el modelo es ingreso de los países; que es una proxy del tamaño de la economía (PIB).

\section{Conclusiones}

El sector textil-confecciones es importante para el sector manufacturero en Colombia; por su aporte al empleo, al PIB nacional y su contribución a las exportaciones. El sector pertenece a un mercado oligopólico y la aglomeración de las empresas cerca a los mercados nacionales le permite tener economías de escala a nivel nacional.

Por otro lado, la teoría económica referente al comercio internacional presenta cambios en el sentido que se desarrollan nuevos entornos económicos, relaciones 
internacionales, tecnologías, estrategias de Estado y costes de trasportes. Para el caso de Colombia existen factores que no permiten ventajas en el comercio internacional, como: los costes de transportes; en el sentido de que los grandes mercados nacionales se encuentran lejos de los puertos marítimos internacionales, tecnología obsoleta y alto coste de la materia prima que es importada.

Además, políticas estratégicas por parte de los países asiáticos en que el Estado favorece al sector, hacen difícil competir con estos países y la hacen vulnerable en el mercado interno y externo. Los niveles de exportaciones del sector en Colombia y del Atlántico mostraron tasas de crecimiento positivas, con excepción de los años 2008 y 2009, debido a la crisis económica en ese periodo y el cierre de relaciones comerciales con Venezuela. En los acuerdos bilaterales establecidos se encontró preferencias arancelarias a los productos originarios de Colombia, y ciertas restricciones a los productos fabricados con materias primas importadas; parece que para Colombia los acuerdos comerciales generan incentivos para exportar; pero para el Atlántico, donde que se encuentra el puerto marítimo de Barranquilla, no es relevante.

El modelo econométrico de crecimiento muestra que la mayor parte de los socios comerciales del Atlántico que resultaron significativos se encuentran cerca del puerto de Barranquilla, es decir, la cercanía a los socios comerciales es un incentivo para exportar por el bajo costo de transporte; encontrándose una relación positiva entre posición geográfica y comercio internacional, lo mismo sucede para Colombia; donde la distancia es un incentivo negativo para las exportaciones Paul Krugman (1991). Otros de los factores que hacen competitiva a la industria es el PIB del socio comercial, y factores comunes al socio como es el idioma y que compartan el mismo país colonizador.

\section{Referencias}

Acuerdo Colombia Estados Unidos. Recuperado de http://www.sice.oas.org/Trade/ COL_USA_TPA_s/COL_USAind_s.asp

Acuerdo Colombia - México. Recuperado de http://www.sice.oas.org/trade/go3/ g3indice.asp

Acuerdo Colombia - Chile. Recuperado de https://www.mincomercio.gov.co/tlc/ publicaciones.php?id=11952

Acuerdo de Libre Comercio Colombia-Canadá. Recuperado de https://www. mincomercio.gov.co/tlc/publicaciones.php?id=16157

Bacaria, J., Osorio, M., \& Artal, A. (2013). Evaluación del Acuerdo de Libre Comercio México-Unión Europea mediante un modelo gravitacional. Economía Mexicana. Nueva Época, (1), 143-163. 
Cárdenas, M., García, C. (2004). El modelo gravitacional y el TLC entre Colombia y Estados Unidos. Recuperado de http://www.repository.fedesarrollo.org.co/ bitstream/11445/947/1/Co_Eco_Junio_2005_Cardenas.pdf

Castro, F., Contreras, D., Tamayo, L., \& Trujillo, L. (2013). Análisis de la competitividad de la cadena algodón, fibras, textiles y confecciones. Recuperado de http://www.repository.fedesarrollo.org.co/bitstream/11445/204/1/ $\mathrm{An} \% \mathrm{C} 3 \%$ A1lisis-de-la-competitividad-de-la-cadena-algod $\% \mathrm{C} 3 \% \mathrm{~B} 3 n$-InformeFinal-Conalgod\%C3\%B3n-_paginaweb.pdf.

Condo, A., Jenkins, M., Figueroa, L., Obando, L., Morales, L., \& Reyes, L. (2004). El sector textil exportador latinoamericano ante la liberalización del comercio. CLACDS-INCAE, processed. Recuperado de http://www.hacienda. go.cr/centro/datos/Articulo/El\%20Sector\%20textil\%20exportador\%20L.A.pdf

Departamento Administrativo Nacional de Estadística - DANE. http://www.dane. gov.co/

Díaz, V. (2012). ¿Se cumple la teoría neoclásica del comercio internacional?: el caso colombiano entre 1980 y 2007. (Doctoral dissertation). Recuperado de http:// bibliotecadigital.univalle.edu.co/bitstream/10893/3669/4/CB-0449733.pdf

Heckscher, E. F. (1968). The effect of foreign trade on the distribution of income. Readings in International Economics. (Editado por Richard E. Caves y Harry G. Johnson. Homewood). (Edición original en sueco, 1919).

Jiménez, V., Martínez, M., Mora, J., \& Martínez, A. (noviembre - diciembre, 2004). El acercamiento comercial: un estudio de los tratados de libre comercio de México. Agrociencia (38). Recuperado de http://www.redalyc.org/articulo. oa? id=30238612\#

Krugman, P. (1979). Increasing returns, monopolistic competition, and international trade. Journal of International Economics, 9(4), 469-479.

Krugman, P. (Ed.). (1986). Strategic trade policy and the new international economics. Mit Press.

Krugman, P. (1992). Geografía y comercio. Antoni Bosch Editor.

Krugman, P., \& Obstfeld M. (2006). Economía internacional: teoría y política. (6 ${ }^{\mathrm{a}}$ ed.). Pearson Education International. Estados Unidos.

Latruffe, L. (2010). Competitiveness, productivity and efficiency in the agricultura and agry-food sectors. OECD Food. Agriculture and Fisheries Papers $\mathrm{N}^{\mathrm{o}} 30$, Publishing, Paris.

López, D., \& Muñoz, F. (2008). Los modelos de gravedad en América Latina: el caso de Chile y México. Comercio Exterior, 58(11), 803-813. 
Ohlin, B. (1933). Interregional and international trade. Cambridge: Harvard University Press.

Oral, M., Kettani, O., \& Yolalan, R. (1992). An empirical study on analyzing the productivity of bank branches. Iie Transactions, 24(5), 166-176.

Pöyhönen, P. (1963). A tentative model for the volume of trade between countries. Weltwirtschaftliches Archiv, 93-100.

Ricardo, D. (1975). Principios de Economía Política y de Tributación. Sraffa, P. (Ed.).

Spencer, J., \& Brander, A. (1983). International r\&d rivalry and industrial strategy. The Review of Economic Studies, 50(4), 707-722.

Tinbergen, J. (1962). Shaping the world economy; suggestions for an international economic policy. 